\title{
STRUCTURAL CHANGES IN THE SYSTEM OF ELECTRON STATES IN A SHEAR-DEFORMED LAYERED CRYSTAL
}

\begin{abstract}
Changes in the structure of electron states in a layered crystal induced by a deformation giving rise to a relative shift of the layers have been analyzed. It is shown that if the deformation force has a low-frequency harmonic component, the period of the deformed lattice changes discretely, and the energy degeneration of the electron states becomes partially eliminated. A space-time deformation removes the energy degeneration for states, whose wave vectors have a component normal to the layer plane and equal to the component of the reciprocal lattice period in the deformed crystal along this direction. It also generates a discontinuity in the functional dependence of the energy on this component. Within a separate time period of perturbation, the energy degeneration becomes eliminated at different time moments, at which the lattice period along the shear direction is a multiple of the shift. The energies within this time interval can be identified with the use of a radio equipment, by detecting the time moments, when the density of electronic states drastically changes.
\end{abstract}

Ke ywords: piezophotoconductivity, layered crystals, shear deformation, periodicity change, layer shift.

\section{Introduction}

Layers as structural elements of a crystalline structure in layered crystals are responsible for certain features in the electronic properties of those materials $[1,2]$. They distinctly manifest themselves in the form of a considerable anisotropy and a field dependence in the electron transfer phenomena and as a structure of interband and phonon absorption spectra in the optical properties. The role of layers-as structural elements that generate an adequate spatial profile of the potential-in the formation of specific features of electronic properties depends on the layer structure and the interaction between the layers. A separate layer in those crystals may possess a complicated multilayer structure, which is formed due to the intense interaction between the layer atoms, so that the layer

(C) Y.M. STAKHIRA, R.Y. STAKHIRA, 2017

1024 becomes somewhat isolated. This property reveals itself in the residual interaction between neighbor layers and in the preservation of their structure in a wide interval of external actions. In particular, it manifests itself in the results of the action of external deformation forces on layered crystals [3].

Characteristic modifications in the system of electron states in layered crystals that arise due to the deformation of corresponding materials are associated with changes of the interaction between the layers occurring as a result of the distance variation between the layers or their relative shift $[4,5]$. In this work, we analyze deformation-induced changes in the structure of electron states and their dynamics, when the layers become shifted with respect to one another (the shear deformation). This is a continuation of works [6-8], in which a mechanism of spectrum formation was proposed for the photoconductivity component

ISSN 2071-0194. Ukr. J. Phys. 2017. Vol. 62, No. 12 
that is proportional to the product of the light intensity and the mechanical stress. A substantial nonmonotonic dependence of the phase difference between the piezophotoconductivity current and the deformation force on the energy of interband transitions, which was found in those works, as well as the conditions for its realization, has to be clarified.

\section{Period of Potential Perturbation in a Shear-Deformed Layered Crystal}

The crystal deformation, by changing the equilibrium coordinates of particles, gives rise to certain modifications in the crystalline structure and to corresponding changes in the crystal potential field. The layered crystals can be deformed by shifting the neighbor layers and leaving the structure of the latter intact, which results in a violation of the lattice periodicity and modifies the interaction between neighbor layers. Such a deformation moves considerable crystal sections by the same distance in the given direction, which finds itself in the coordinate dependence of the potential. As a rule, the layer shift is achieved at insignificant efforts, which enables such variations of the potential to be considered as perturbations. The main controlled quantity of such a perturbation is its period.

Let us consider structural variations in a layered crystal subjected to a force action. The force is the sum of a constant, $F_{c}$, and a low-frequency harmonic, $F_{s} \sin \Omega t$, component. If the deforming force has a component in the layer plane, it can bring the neighbor layers closer to each other and relatively shift them. Let us confine the consideration to the case where the deforming force insignificantly modifies the layer structure and the distance between the layers. The crystal deformation under the action of such a force does not change the lattice period in the layer plane, $(x, y)$, but it modifies the lattice period in the direction normal to the planes. Owing to the preservation of structure identity in the course of a shear deformation, the lattice period in this direction can change only discretely with a minimum step equal to the layer thickness $d_{z}^{0}$. This circumstance gives rise to a complicated dependence of the lattice period $d_{z}$ in the deformed crystal on the relative shift $L$, which changes continuously.

If the layers are shifted in the direction, in which the lattice period in the layer plane equals $d_{x y}$, the identical spatial arrangement of two neighbor layers in the crystalline structure is reached, when their total realtive shift is the smallest multiple of $d_{x y}$. A required condition for this equality to be satisfied is the multiplicity relationship between the quantity $N d_{x y}$ and the relative shift $L$,

$N d_{x y}=n L$,

where $n$ is the number of sheared layers, and $N$ is the smallest integer number, at which $n$ is an integer for the given $d_{x y}$ - and $L$-values. Hence, the lattice period in the deformed crystal in the direction normal to the layer plane is determined by the number of shifted layers, $d_{z}=n d_{z}^{0}$. If the lattice period $d_{x y}$ is a multiple of the shear magnitude (this requirement is satisfied for shifts $L \leq \frac{d_{x y}}{2}$ ), the realized $n$-values are small, so that the values of the period $d_{z}$ are the smallest.

The value of the period $d_{z}$ in the deformed crystal and in the corresponding deformation-induced perturbation of the crystal potential can be changed by one layer thickness owing to an additional shift. If the period $d_{z}$ is obtained at the shift $L_{c}$, its increase by one layer thickness can be reached by reducing the shift by $\Delta L_{+}=-L_{c} \frac{1}{n+1}$. This period can be reduced by increasing the shift $L_{c}$ by $\Delta L_{-}=L_{c} \frac{1}{n-1}$. The reduction of the period $d_{z}$ by an integer number $p$ of the layer thickness can be obtained, if the shift $L_{c}$ is increased by the additional shift $\Delta L_{-}=L_{c} \frac{p}{n-p}$, whereas its increase by the number $p$ of layers is reached by reducing the shift $L_{c}$ by the value of $\Delta L_{+}=-L_{c} \frac{p}{n+p}$, provided that $p \leq n-1$.

Therefore, the period of a deformation-induced perturbation of the crystal potential is determined not only by the shear magnitude, but also by its relation to the period $d_{x y}$. The multiplicity relationship between the quantity $N d_{x y}$ and the shift $L$ is a necessary condition for the layer structure preservation, when the period of a potential perturbation changes. The dependence of the perturbation period on the shift is given by the multiplicity factor $n$, which is determined by the values of a shift and the period $N d_{x y}$ of the lattice, in which the shift takes place. The multiplicity ratio between the period $d_{x y}$ and the shift magnitude is equal to the number of sheared layers, whose total thickness is equal to the crystal period $d_{z}$ in the direction normal to the layer plane. A change of the multiplicity factor by one corresponds to the change of this period by the thickness of one layer. Below, we 
confine the consideration to shifts $L \leq \frac{d_{x y}}{2}$, at which the multiplicity factor values are small.

\section{Time Evolution of Deformation-Induced Potential Perturbation}

Specific features of the processes responsible for the formation of the periods of a potential perturbation at the shear deformation distinctly manifest themselves in the temporal dependences of their parameters. In the case of layered crystals, for which the relaxation time $\tau$ of mechanical stresses is rather short, and provided that the frequency $\Omega$ of the harmonic component is so low that $\frac{2 \pi}{\Omega} \gg \tau$, the main factor giving rise to time variations in the deformationinduced perturbation is the time dependence of the relative shift between the layers. The harmonic force component makes the shift $L$ time-dependent, $L=$ $=L_{c}+L_{s} \sin \Omega t$, so that $L$ changes within the interval $\left[L_{c}-L_{s}, L_{c}+L_{s}\right]$ depending on the constant force component $L_{c}$ and the amplitude $L_{s}$ of the harmonic component. Within this interval, the shift $L$ changes continuously (increasing or decreasing, depending on the deforming force phase) and inphase with the harmonic phase. Under certain conditions, at definite time moments $t_{p}$ within a temporal period of the harmonic component, the period $d_{x y}$ becomes a multiple of the shift $L$. As a result, the smallest multiplicity factor $n$ and the perturbation period $d_{z}$ become time-dependent.

The time moments, at which the multiplicity ratio between the period $d_{x y}$ and the shift $L$ changes, can take place only as a result of the additional shifts $\Delta L$, with the harmonic part of the shift playing this role in our case. The harmonic component of a shift, being regarded as an additional shift to $L_{c}$ (considered to have a given constant value), can result in its reduction or growth by an integer number of $p$-values, depending on the harmonic phase. In the case of harmonic increments, $\Delta L=L_{s} \sin \Omega t$, that diminish $L_{c}$ by the smallest value $\Delta L_{+}=-L_{c} \frac{p}{n+p}$, the multiplicity factor $n$ grows to $n+p$. If the harmonic component makes the shift $L_{c}$ larger by $\Delta L_{-}=L_{c} \frac{p}{n-p}$, the multiplicity factor $n$ decreases to $n-p$, where $p=1,2, \ldots$.

The harmonic component of the total shift is a direct origin of temporal variations of the period $d_{x x}$ in the deformed crystal. It plays the role of an additional shift to the given constant shift $L_{c}$. The harmonic component, by changing the total shift from
$L_{c}-L_{s}$ to $L_{c}+L_{s}$, creates total shifts that are multiples of the period $d_{x y}$. Within the harmonic period, every such shift $L_{p}=L_{c}+L_{s} \sin \Omega t_{p}$ is associated with a specific multiplicity factor $n_{p}$ and a specific time moment of its creation.

The multiplicity factor $n=\frac{d_{x y}}{L_{c}}$ associated with the given constant shift $L_{c}$ can be either reduced by the harmonic shift component by an integer number of $p$-values, if the harmonic component at the time moment $t_{p}^{-}$is equal to the shift $\Delta L_{-}$,

$L_{s} \sin \Omega t_{p}^{-}=L_{c} \frac{p}{n-p}$,

or increased by an integer number of $p$-values in the case of the equality

$L_{s} \sin \Omega t_{p}^{+}=L_{c} \frac{p}{n+p}$,

which is obeyed in corresponding harmonic intervals.

Owing to the continuous growth of the total shift $L$ within the harmonic phase interval $0 \leq \varphi_{1} \leq \pi / 2$, the multiplicity factor diminishes discretely at the time moments

$t_{p}^{-}=\frac{1}{\Omega} \arcsin \left(R \frac{p}{n-p}\right)$

reckoned from the time reference point $\Omega t_{0}=0$, $R=\frac{L_{s}}{L_{c}}$. The maximum number of the multiplicity factor changes, $p_{1}$, corresponds to the total shift $L=L_{c}+L_{s}$ and is equal to the largest integer part of the fraction $p_{1}=\frac{n}{R+1}$. The number of $p_{1}$-time moments, at which the multiplicity factor changes, depends on its value at the time moment $t_{p}^{-}=0$. The multiplicity factor $n=2$ cannot be diminished by any layer shift.

Within the time intervals corresponding to the harmonic phases $\pi / 2 \leq \varphi_{2} \leq \pi$, the multiplicity factor, being reduced to $n-p$, diminishes discretely due to the harmonic reduction of the total shift from $L_{c} \frac{n}{n-p_{1}}$ to $L_{c}$. The time moments $t_{p}^{+}$, at which the considered multiplicity factor changes by one take place, when the harmonic component acquires values that are equal to the corresponding increments $\Delta L_{+}$, which increase the multiplicity factor,

$t_{p}^{+}=\frac{\pi}{2 \Omega}+\frac{1}{\Omega} \arcsin \left(R \frac{p}{n-p_{1}+p}\right)$.

Similar variations of the multiplicity factor $n$ occur in the next half-period of a harmonic. Within the

ISSN 2071-0194. Ukr. J. Phys. 2017. Vol. 62, No. 12 
interval of the harmonic phase change from $\pi$ to $\frac{3}{2} \pi$, due to a reduction of the total shift, the multiplicity factor drastically increases to $n+p_{1}$. The maximum increment of the multiplicity factor, $p_{1}$, is equal to the largest integer part of the fraction $p_{1}=\frac{R n}{1-R}$. The time moments $t_{p}^{+}$, at which the multiplicity factor changes, take place, when the harmonic shift component is equal to one of the increments $\Delta L_{+}$giving rise to the multiplicity factor growth by one.

Within the time interval corresponding to the $\varphi_{4}$ phase interval from $\frac{3}{2} \pi$ tp $2 \pi$, when the shift increases from $L_{c} \frac{n}{n+p_{1}}$ to $L_{c}$, the multiplicity factor decreases from $n+p_{1}$ to $n$ at the time moments $t_{p}^{-}$that satisfy a dependence for additional shifts $\Delta L_{-}$, which is similar to Eq. (5).

Thus, the harmonic component of the relative layer shift makes the component of the crystal lattice period oriented normally to the layer plane timedependent. The time dependence of the period $d_{z}$ in this direction is governed by discrete shifts of strictly given quantities in the form of sudden changes of its value by a value of the layer thickness at the time moments corresponding to the phase values of the harmonic components of those shifts. Within the time intervals, when the period changes by one layer thickness, the lattice period in the deformed crystal remains constant. The duration of those intervals, which are responsible for the time dependence of the period $d_{z}$, substantially changes in the harmonic phase intervals $0<\varphi<\pi$ and $\pi<\varphi<2 \pi$.

The time dependence of the component $d_{z}$ of the crystal lattice period is a complicated discrete nonharmonic periodic function, in which an antiphase tendency with respect to the harmonic phase is conditionally preserved. In Fig. 1, the time diagrams for the parameter set $L_{c}=0.25, L_{s}=\frac{1}{2} 0.25$, and $d_{x y}=1$ are shown.

\section{Deformation-Induced Changes in the Structure of Electron States}

The deformation of a layered crystal leads to certain modifications in its crystal structure. As a result, the coordinate dependence of the crystal field potential undergoes corresponding changes. In the case of a deformation giving rise to a relative shift of neighbor layers, the microscopic fragments of a crystal structure may move over rather long distances, which can bring about substantial modifications in the structure

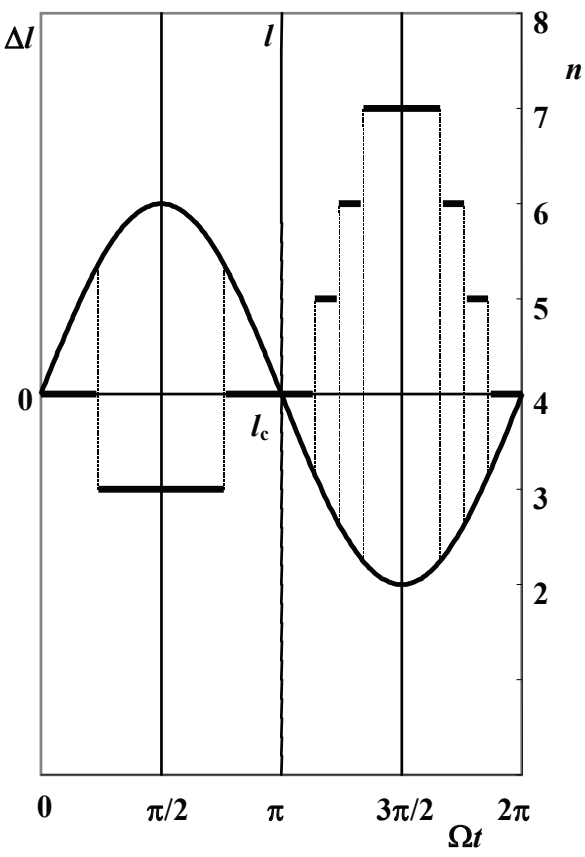

Time diagram for the parameter set $L_{c}=0.25, L_{s}=\frac{1}{2} 0.25$, and $d_{x y}=1$

of electron states. The shear deformation of a layered crystal is obtained, as a rule, at insignificant external forces and, therefore, can be considered as an origin of the deformation-induced potential perturbation.

The deformation-induced perturbation of the crystal potential is generated by a relative shift of layers and substantial changes in their interaction. The shift between the layers changes the distance between the identical structural elements in the neighbor layers and, accordingly, affects both the interaction between them and the dependence of this interaction on the layer structure. The changes that are induced in the weak interaction between the layers by their relative shift, at which the layered structure of a deformed crystal and the structure of the layers themselves survive, stimulate changes in the crystal potential, which are considered as perturbations. If the deforming force has a low-frequency harmonic component, which provides a quasistationary regime of the process, the period of this perturbation may depend on the shift between the neighbor layers, being a complicated discrete function of the time.

The relative shift between the neighbor layers takes place synchronously with the harmonic phase. At the same time, the perturbation period changes only at 
definite discrete shift values arising at the corresponding time moments within the harmonic period. Such a temporal dependence of the perturbation allows its influence on the electron states to be considered in the adiabatic approximation of perturbation theory. In the framework of this approximation, the harmonic component of a perturbation is considered to be a slowly varying background for short, in comparison with the harmonic period, time intervals with the varying perturbation periodicity regarded as a rapid component in the temporal dependence of the perturbation.

The deformation of a crystal by the total harmonic force gives rise to a shift of layers, which oscillates inphase with the phase of the harmonic force component. The cophasal behavior of this harmonic and the period of the deformation-induced perturbation becomes lost because of a complicated non-harmonic time dependence of the perturbation period.

Changes in the perturbation period realized in the form of sudden increments of its value takes place only at the time moments, when the shift values and the shift period $d_{x y}$ are multiple to each other. In particular, the period of perturbation created by the constant force component, $d_{z_{c}}=\frac{d_{x y}}{L_{c}} d_{z}^{0}=n_{c} d_{z}^{0}$, diminishes by the harmonic component due to the total shift growth in the time interval from $\Omega t_{0}=0$ to $\Omega t_{1}=\pi$ and increases in the time interval corresponding to the harmonic phase difference from $\pi$ to $2 \pi$. With respect to the time reference point, $t_{0}=0$, at which the shift equals $L_{c}$, the period of the deformation-induced perturbation changes in antiphase to the harmonic phase.

Hence, the shear deformation of layers results in the formation of a set of short-lived Bragg planes, which coincide with the layer planes. Their energy position and the time of emergence are determined by the component of the period in the reciprocal lattice, $q=\frac{2 \pi}{d_{z}}$. At the corresponding time moments, owing to the diffraction of Bloch waves at those planes, there emerge discontinuities in the energy dependence of electron states $\varepsilon(\mathbf{k})$ at all wave vectors $\mathbf{k}$, whose $k_{z}$ component equals the vector component $q=\frac{2 \pi}{d_{z}}$. The comparability of the $q$-value and the $k_{z^{-}}$ components of the wave vectors in the Brillouin zone allows such $\varepsilon(\mathbf{k})$-energy discontinuities to be realized in the whole interval of the conduction band. For a given, fixed in time, perturbation period $d_{z}$, the discontinuities in the energy dependences appear simul- taneously at all energies, for which the corresponding wave vectors have the $k_{z}$-component $k_{z}=\frac{2 \pi}{d_{z}} m$, where $m=1,2,3 \ldots$ In the vicinities of those energies, the density of electron states drastically changes, which results in the corresponding modifications of electronic properties of the crystal. The energy bands that arise owing to the Bragg diffraction of Bloch waves are formed at time moments that unambiguously correspond to the component $q$ and can be identified by its magnitude.

The elimination of the $\varepsilon(\mathbf{k})$-energy degeneration by means of a deformation-induced perturbation gives rise to adequate changes in the structure of electron states in the undeformed crystal. Owing to changes in the coordinate dependence of the crystal potential and its symmetry, the shift of the layers removes the degeneration at energies corresponding to the wave vectors with the $k_{z}$-component equal to the component of the perturbation vector $q$. The elimination of the state degeneration for the given vector $q$ takes place simultaneously for all energies with $k_{z}=q$ at the corresponding time moment. As a result, the energy dispersion curve becomes discontinuous at wave vectors with the $k_{z}$-component $k_{z}=q$, and the corresponding electronic properties of the crystal change. The degeneration elimination dynamics and its influence on the structure of electron states completely coincide with the results of the Bragg diffraction of Bloch waves.

The shear deformation of layers preserves the structure and the major physical parameters of a crystal, except for a change of the crystal lattice period in the direction normal to the layer plane, which takes place discretely by one layer thickness. This model makes it possible to compare variations arising in the structure of electron states in the crystals, for which the $d_{z^{-}}$ components of the lattice periods differ by an integer number of times. Provided that the volume of a deformed crystal remains constant, this deformation results in multiple changes of the unit cell volume in the reciprocal lattice and in the corresponding changes for the number of allowed wave-vector values. Every fixed period of the deformed crystal corresponds to a specific spatial distribution of structural units, the corresponding dependence on the layer structure, and the interaction between the layers. Among various changes in the structure of electron states induced by the layer shift, the discontinuity in the functional dependence of the energy on the wave vector and the 
time of its realization remain invariant for the considered model of deformed crystal.

The structural changes in the energies of electron states associated with the layer shift controlled by deforming forces can be found experimentally from the time dependence of the harmonic component of the monochromatic light absorption factor, which plays the role of a monitor, within a single harmonic period. In works [7, 8], such changes were estimated from the spectral dependence of the phase shift of the conductivity component that is proportional to the product of the light intensity and the mechanical stress with respect to the phase of a modulating force.

\section{Conclusions}

The deformation of a layered crystal realized in the form of a relative shift between the neighbor layers changes the component of the crystal lattice period in the direction perpendicular to the layer plane. The lattice period changes by an integer number of layer thicknesses at specific shift values, for which the lattice period in the shift direction is a multiple. The shifts induced by a force with a low-frequency harmonic component result in a non-harmonic discrete dependence of the lattice period on the time within the force harmonic period. The time moments of the lattice-period change unambiguously correspond to the shift magnitudes and the multiplicity factor of the period in the shift direction.

The deformation-induced space-time perturbation of the crystal potential, which is created by shifting the layers, when being considered in the adiabatic approximation, results in a partial elimination of the energy state degeneration and the appearance of discontinuities in the energy dependence on the wave vector component normal to the layer plane. The partial elimination of the electron energy degeneration takes place at the time moments $t_{p}$, when the components of the wave vector $k_{z}$ equal

$k_{z}=\frac{2 \pi}{d_{z}^{0}} \frac{L_{c}+L_{s} \sin \Omega t_{p}}{d_{x y}}$.

Hence, the degenerated energy can be identified by the moments of its degeneration. Those moments coincide with the moments, when the electronic properties of the crystal drastically change due to substantial changes in the electron density of states in the vicinities of degenerated energies.
Thus, the energies of electronic states, whose degeneration can be partially eliminated by a controlled deformation-induced perturbation of the crystal lattice, can be identified by the time moments, when this elimination takes place. The elimination of the degeneration of a specific energy state takes place at a characteristic time moment and is accompanied by a drastic change in the electron density of states in the vicinity of this energy. Accordingly, the moment, when the degeneration becomes eliminated, can be identified by detecting the change of electronic properties.

In works [8, 9], the energy dependence of the time moments, when the energy degeneration of electronic states in the $\mathrm{In}_{4} \mathrm{Se}_{3}$ layered crystals was eliminated, was estimated from the spectral dependence of the phase shift between the harmonic and the nonharmonic component of the piezophotocurrent. The structural modifications in the system of electron states in layered crystals that arise due to the shear deformation of layers can be efficietly used. In particular, this effect can be applied to analyze light spectra within radio engineering methods and taking no advantage of dispersing elements [10].

1. G.L. Belen'kii, E.Yu. Salaev, R.A. Suleimanov. Deformation phenomena in layered crystals. Usp. Fiz. Nauk 155, 89 (1988) (in Russian).

2. D.M. Bercha, K. Rushchanskii, I.V. Slipukhina, I.V. Bercha. Manifestation of deformation effect in band spectra in crystals with inhomogeneous bonding. Cond. Matter Phys. 6, 229 (2003).

3. M.S. Brodin, I.V. Blonskii, Excitonic Processes in Layered Crystals (Naukova Dumka, 1986) (in Russian).

4. R.Y. Stakhira, G.D. Danylyuk, Ya.M. Buzhuk. Piezoohotoconductivity spectra and X-ray structural study of layered structures. Novi Tekhnol. 27, 62 (2010) (in Ukrainian).

5. I.V. Slipukhina, L.Yu. Kharkhalis, I.V. Bercha. Influence of shear deformations and interstitial impurities on the dispersion laws for current carriers in $\mathrm{In}_{4} \mathrm{Se}_{3}$ layered crystals. Nauk. Visn. Uzhgorod. Univ. 19, 14 (2006) (in Ukrainian).

6. Y.M. Stakhira, R.Y. Stakhira. Piezophotoconductivity spectra in monopolar semiconductors. Zh. Fiz. Dosl. 2, 376 (1998) (in Ukrainian).

7. J.M. Stakhira, R.J. Stakhira, V.P. Savchyn. Concentration mechanism of piezophotoconductivity. Ukr. J. Phys. 50, 588 (2005).

8. Y. Stakhira, R. Stakhira. Structure of spectra of timedependent piezophotoconductivity. J. Phys. Stud. 11, 215 (2007).

9. Y. Stakhira, R. Stakhira. Mechanism of non-stationary piezophotoconductivity spectrum formation in layered crystals. Ukr. J. Phys. 58, 1159 (2013). 
10. Y.M. Stakhira, R.Y. Stakhira, V.M. Belyukh. Patent Ukraine No. 108674 (2016) (in Ukrainian).

Received 28.07.17. Translated from Ukrainian by O.I. Voitenko

Й.M. Сmaxipa, Р.Й. Сmaxipa

СТРУКТУРНІ ЗМІНИ У СИСТЕМІ

ЕЛЕКТРОННИХ СТАНІВ ШАРУВАТОГО КРИСТАЛА, ДЕФОРМОВАНОГО ЗСУВОМ ШАРІВ

Р е $з$ ю м е

Проаналізовано зміни структури електронних станів шаруватих кристалів, які зумовлені деформацією кристала, що приводить до відносного зсуву шарів. Показано, що та- ка деформація силою, яка має гармонічну складову низької частоти, приводить до дискретної зміни періоду гратки і часткового зняття виродження енергій електронних станів. Просторово-часове деформаційне збурення приводить до зняття виродження енергій, хвильові вектори яких мають компоненту, нормальну до площини шарів і рівну компоненті періоду оберненої гратки деформованого кристала у цьому напрямку, і розриву функціональної залежності енергї від такої компоненти. У межах окремого періоду зняття виродження енергії відбувається в різні ексклюзивні моменти часу, в які період гратки у напряму зсуву є кратним величинам зсуву. Енергї цього інтервалу можуть бути ідентифіковані радіотехнічними методами за моментами часу різкої зміни густини електронних станів. 